\title{
Pixel area variation in CCDs and implications for precision photometry
}

Roger M. Smith, Gustavo Rahmer

Roger M. Smith, Gustavo Rahmer, "Pixel area variation in CCDs and implications for precision photometry," Proc. SPIE 7021, High Energy, Optical, and Infrared Detectors for Astronomy III, 70212A (22 July 2008); doi: 10.1117/12.789665

Event: SPIE Astronomical Telescopes + Instrumentation, 2008, Marseille, France 


\title{
Pixel area variation in CCDs and implications for precision photometry \\ Roger M. Smith ${ }^{* a}$, Gustavo Rahmer ${ }^{\mathrm{a}}$ \\ ${ }^{a}$ Caltech Optical Observatiories, California Institute of Technology, MC105-24, 1200 E. California Blvd, CA 91125, USA
}

\begin{abstract}
Images with smooth and moderately flat illumination are commonly used to calibrate pixel to pixel sensitivity variation without consideration that some structure on short spatial scales may be due to reallocation of area between pixels. Errors in the position of pixel boundaries have the effect of repartitioning charge between pixels but do not affect the total charge collected. Since the resulting errors tend to cancel when combining signal from adjacent pixels, this effect has gone largely unnoticed. However proposed wide field astronomical surveys, which strive to achieve high photometric precision with coarse spatial sampling, must take this into account. We present simple analysis techniques to identify how much flat field structure is due to systematic and random variations in pixel area, rather than sensitivity, as a function of row/column direction and spatial frequency. Analysis of data from CCDs made with radically different technologies and pixel sizes by different manufacturers suggests that pixel size variation in the column direction probably dominates QE variations on short spatial scales for all CCDs. Refinements to flat field calibration methods and tests to confirm their efficacy are proposed.
\end{abstract}

Keywords: flat fielding, pixel size, pixel area, high resistivity, depletion

\section{INTRODUCTION}

Cumulative errors in pixel pitch in CCDs must be very small due to the stringent requirements of mask alignment over large areas, however irregularities during etching or doping can introduce random local offsets in the peak of the barrier potential so that one pixel gains area at the expense of its neighbor. The relative size of potential wells created by parallel clock voltages and the built in irregularities determine the magnitude of the shift in the peak of the barrier potential (Fig. 1). Variation in response to smooth illumination on short spatial scales, commonly referred to as Pixel Response Non-Uniformity (PRNU), can occur even in the absence of QE variations due to reallocation of collecting area between pixels, though in practice some mixture of both effects is usually present.

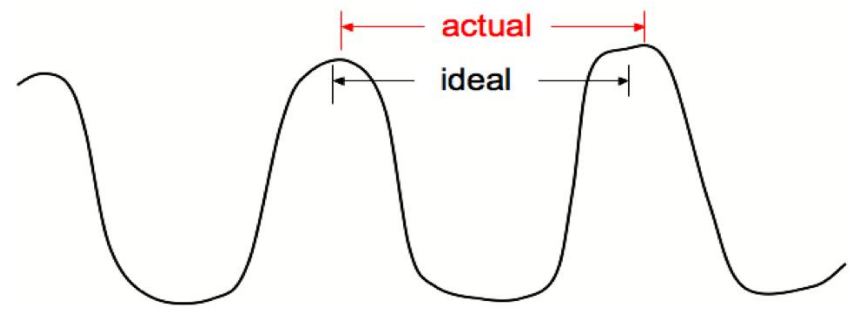

Fig. 1: Built in perturbations to the potential profile along the column, or small defects in the electrode shape will offset the peak of the barrier potential imposed by the clock electrodes. This will repartition collecting area between adjacent pixels, while also shifting the effective center of the pixel slightly.

Although a description of this effect can be found in at least one CCD textbook ${ }^{[1]}$, astronomers typically divide science images by the PRNU image, to correct for what is assumed to be purely a sensitivity variation (Fig. 2). However, changes in the position of a pixel boundary (Fig. 3) only affect the allocation of charge between pixels and not the amount of charge collected. Thus division by the PRNU image followed by summation of the signal will produce an erroneous estimate of the total flux, when pixel boundary offsets are a significant source of PRNU compared to QE variations.

\footnotetext{
*rsmith@astro.caltech.edu; phone 1626 395-8780; fax 1626 568-1517
}

High Energy, Optical, and Infrared Detectors for Astronomy III, edited by David A. Dorn, Andrew D. Holland, Proc. of SPIE Vol. 7021, 70212A, (2008) · 0277-786X/08/\$18 - doi: 10.1117/12.789665 
Since pixels can only increase in size at the expense of their neighbors, the resulting errors will tend to cancel if the object of interest (a star for example) spans several pixels in the direction of the pixel boundary shift. However, wide field surveys in space, such as the SuperNova Acceleration Probe ${ }^{[2]}$ are driven towards coarse sampling of the point spread function (PSF) by a desire to overcome read noise in the shortest possible exposure time (by spreading the light among the fewest possible pixels), and by the desire to obtain the largest possible field of view for a given investment in detectors. Therefore survey missions are generally more vulnerable to this pixel size variation. At the same time SNAP is striving for higher precision photometry, comparable to the amplitude of the PRNU.

SNAP will mitigate this problem by combining multiple exposures at different positions on the CCDs, and, while the sampling of the PSF is coarse in the bluer passbands, the light will still be generally divided among several pixels. Nonetheless it is important to quantify the effect to see how large a term it represents in the photometric error budget. The goal of this investigation is to assess the relative contributions of sensitivity and pixel area variation to the PRNU (Fig. 4), and to identify how further safety margin in photometric accuracy might be gained by changes to the flat fielding algorithm.

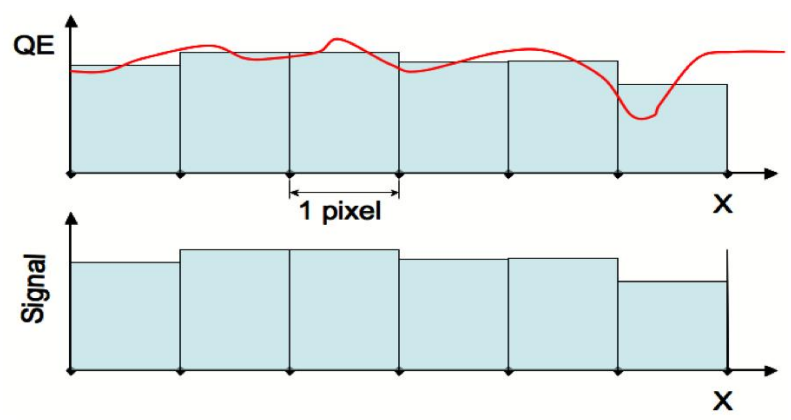

Fig. 2: Conventional wisdom holds that the Pixel Response Non Uniformity is due to Quantum efficiency variation alone.

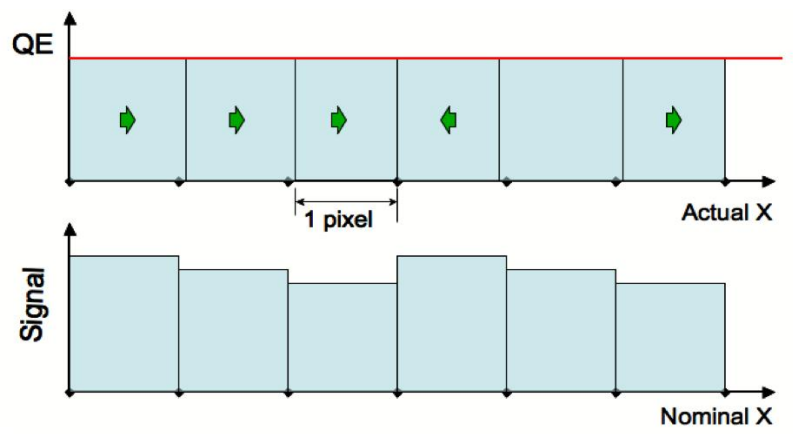

Fig. 3: Offsets in the pixel boundaries from their nominal position will cause non-uniform response to flat illumination in the absence of any QE variation simply due to differences in collecting area between pixels. The positions of pixels are offset as well, which will affect attempts to do photometry by profile fitting.

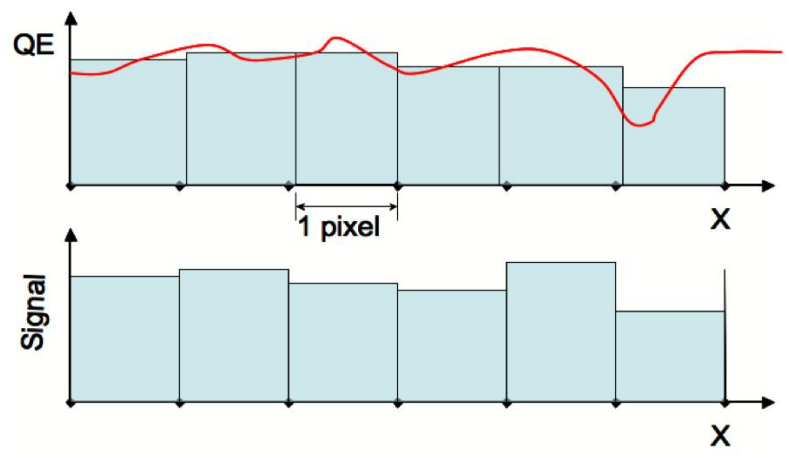

Fig. 4: In reality, Pixel Response Non-Uniformity will be due to some combination of QE and pixel area variations, but applying the PRNU correction directly could do more harm than good if pixel area variation is the dominant effect. 


\section{MEASUREMENT OPTIONS}

The following measurements might be made to detect pixel size variations:

PRNU variation induced by clock voltage change: vary the high level of the parallel clocks, while projecting a flat field on the CCD. Since this only monitors changes in pixel area it serves only to detect the presence of pixel size variations rather than quantify it relative to $\mathrm{QE}$ variation.

Dependence of photometry on pointing (spot scan): vary the position of a simulated star field over a range of (say) 10 pixels in sub pixel increments. After linearity correction, the total signal will respond to sensitivity variations and not pixel size. Comparing scans of high contrast spots or lines to flat illumination might allow pixel area and QE to be disentangled but this requires a specialized projection setup, is difficult to perform over large areas, and cannot be reproduced as part of the calibration process while observing.

Anti-correlations in PRNU: this is the subject of the rest of this paper. This relies on a more complicated line of reasoning, but only requires high signal-to-noise flat fields for analysis.

\section{SAMPLE FLAT FIELDS}

A conventional backside illuminated n-channel e2v CCD with $30 \mu \mathrm{m}$ pixels was chosen for initial tests since it has deep wells and good cosmetics. Twenty flat fields (Fig. 5) each with approximately half a million electrons per pixel were coadded, so that shot noise per pixel was about $0.03 \%$ of the mean, compared to a $0.9 \%$ rms PRNU. The high frequency PRNU structure (Fig. 6) was extracted by dividing the flat field by a 9x9 boxcar-filtered version of itself. This size boxcar was chosen to remove both the illumination gradient in the flats and mid frequency ripples induced by the laser annealing of the boron implant, which are not relevant to this study.

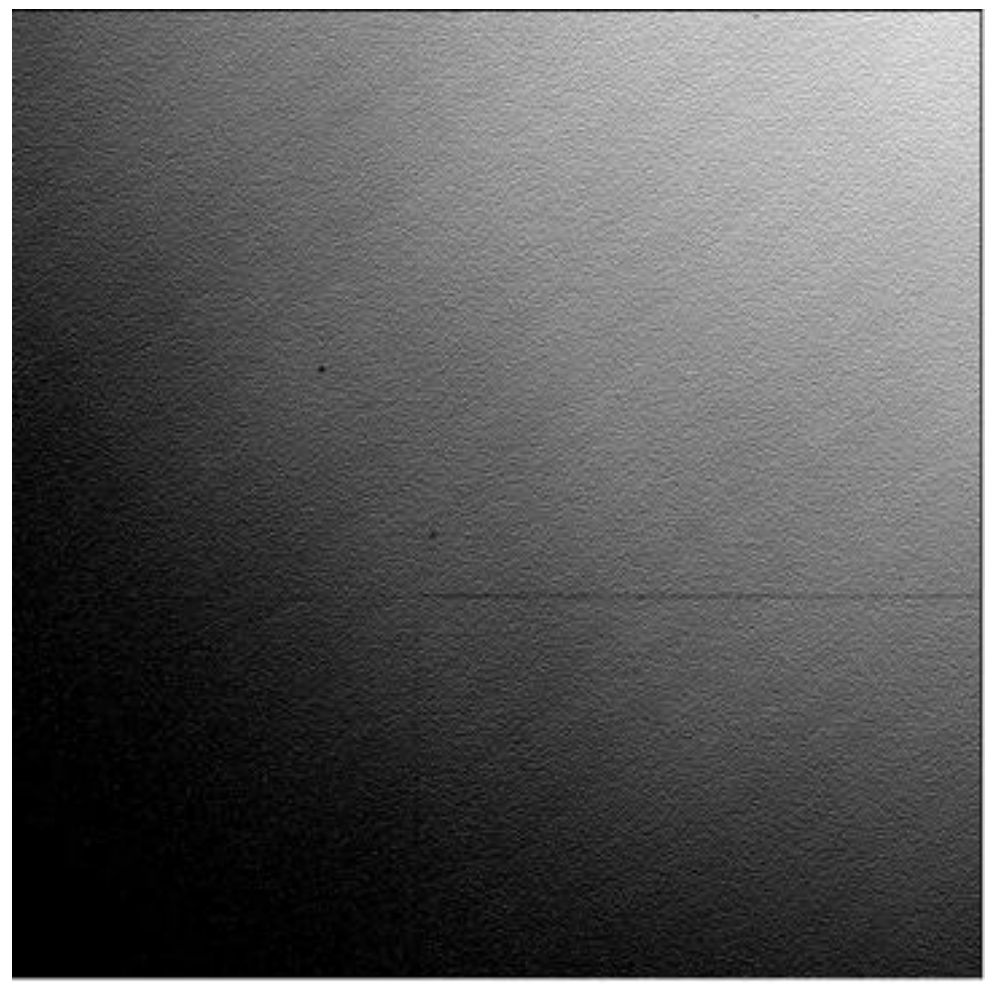

Fig. 5: This 500x500 pixel portion of a raw mid-band flat field was obtained by coadding multiple frames for a total of $\sim 10$ million electrons per pixel and thus a $0.03 \%$ shot noise. It is the granularity at the highest spatial frequencies which is the subject of this study. The relatively large gradient is due to illumination. The faint mid frequency ripples are $\mathrm{QE}$ variations due to laser annealing of the Boron implant, while the two dark spots are probably surface contamination. The faint vertical and horizontal lines are at mask stitching boundaries. 


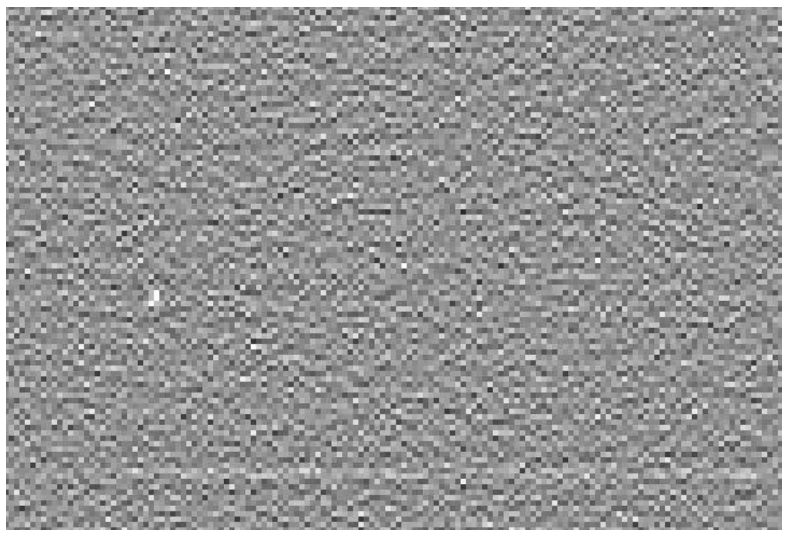

Fig. 6: This $160 \times 90$ pixel section of the PRNU image (white = low sensitivity) shows that most of the CCD delivers very uniform response. QE structure on mid to large spatial scales has been deliberately suppressed along with the illumination gradient by dividing the raw flat by the $9 \mathrm{x} 9$ boxcar filtered flat to produce this PRNU image. The granularity appears random and constitutes only a $0.89 \% \mathrm{rms}$ variation. The small QE drop and stitching line are representative of the very few exceptions to the random granular structure.

\section{PRNU SMOOTHING RATES}

Although inspection of the PRNU image in Fig. 6 provides little clue, evidence of pixel size variation is found in the rate at which pixel to pixel structure is smoothed by averaging lines and columns (Fig. 7).
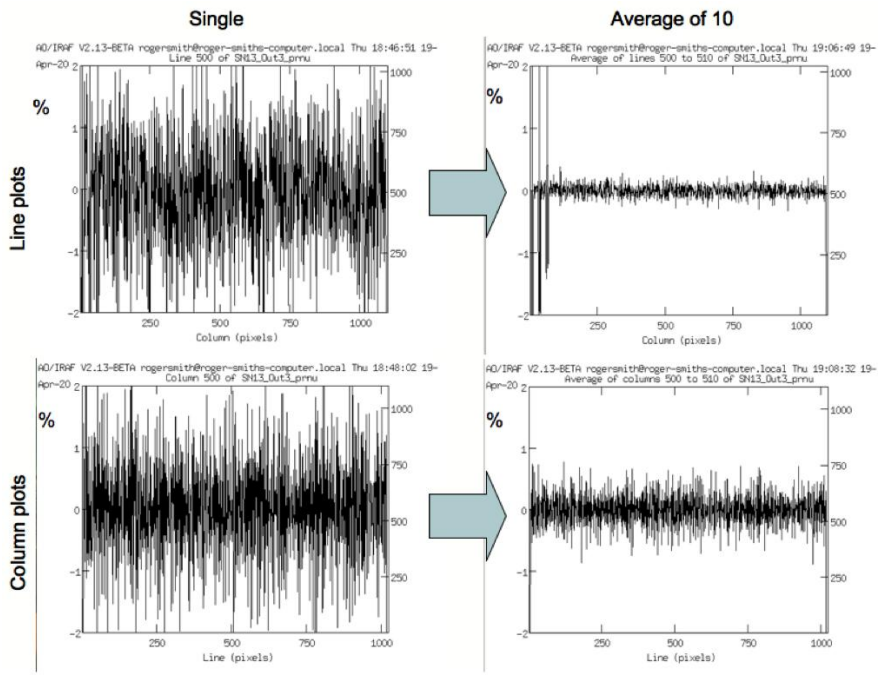

Fig. 7: PRNU plots are similar along single lines or columns (top and bottom left respectively), but the average of 10 lines (top right) and ten columns (bottom right) show markedly different pixel to pixel variation.

If $\mathrm{N}$ rows in the PRNU image are averaged, the standard deviation is reduced linearly as would be expected if the charge is simply being reallocated between pixels. Since a pixel gains area only at the expense of its neighbor, it is only the motion of the boundary at the edge of the box which can affect total area, so the error in total area scales as $1 / \mathrm{N}$, as is observed for the standard deviation of the line averages (i.e., smoothing is along columns).

The standard deviation reaches a floor when sufficient lines have been averaged so that the width variation that correlates along entire columns dominates the random variations. These column width variations are periodic suggesting that they 
are due to errors in the printing of the lithographic masks. This will be discussed below in the context of the SNAP CCDs.

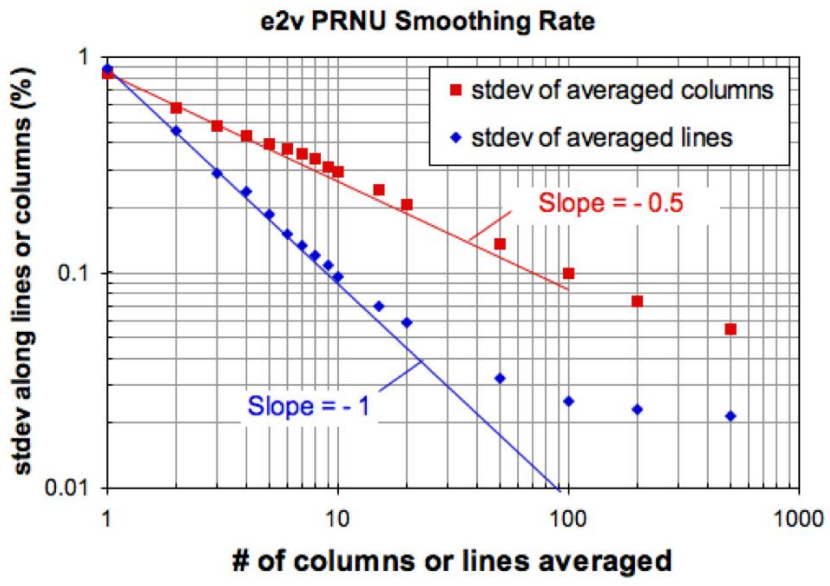

Fig. 8: The standard deviation along lines scales as $1 / \mathrm{N}$ when $\mathrm{N}$ lines are averaged, whereas the standard deviation along columns scales as $1 / \sqrt{ } \mathrm{N}$ when columns are averaged, indicating that the random variations along columns are anticorrelated whereas along lines they are uncorrelated.

Fig. 8 shows very different behavior when averaging $\mathrm{N}$ columns (i.e., smoothing along lines): the reduction of standard deviation as $1 / \sqrt{ } \mathrm{N}$ indicates that PRNU is dominated by random effects, which could be due to sensitivity variation in the direction orthogonal to the smoothing (i.e., along columns).

\section{FOURIER ANALYSIS}

While PRNU smoothing rate provides an easy test for pixel size variation, much more can be learned from the analysis of the spatial power spectra. Through simulation we discovered, to our surprise, that the shape of the power spectrum of the PRNU induced by randomizing the boundary positions is independent of the distribution chosen for the boundary offsets. Fig. 9 shows that Gaussian, flat topped and discrete probability distributions for the boundary offsets produce identical power spectra. The amplitudes of the spectra were found to coincide when the input distributions were scaled to give the same rms pixel size variation, as predicted by Parseval's Theorem. We speculate that the lack of dependence on the type of random distribution is some manifestation of the Central Limit Theorem.

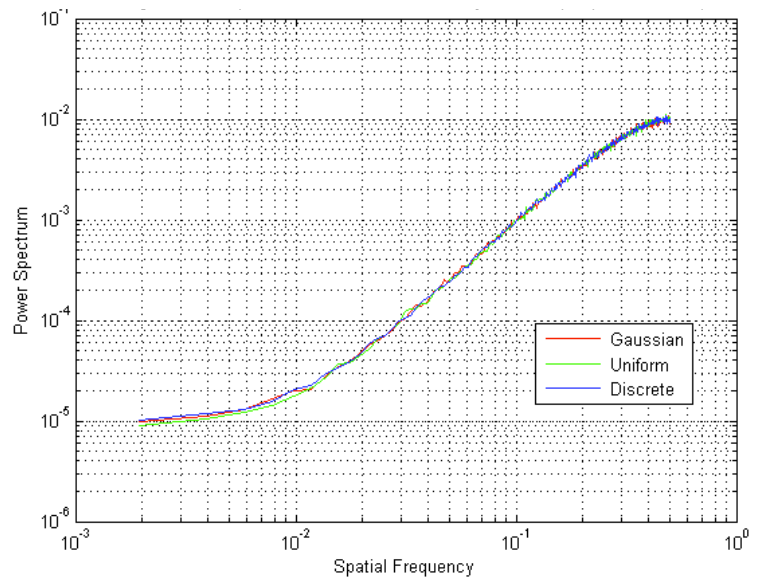

Fig. 9: Spatial power spectra for simulations using Gaussian, random and discrete $(-x, 0,+x)$ distributions. The power spectrum of pixel area variations has the remarkable property that the shape of the spectrum is independent of the distribution used to randomize the pixel boundary offsets. 
Fig. 10 shows the power spectra along lines and column directions for coadded raw flats. The radically different shape in each direction is suggestive there is a pixel size variation along the columns only. Comparison with the simulated pixel size spectrum in Fig. 9 is complicated by the fact that the mid spatial frequencies are swamped by the illumination gradient in the raw flats so that comparison to the simulated spectrum can only be performed at the highest spatial frequencies. By contrast, in Fig. 11 we see that the power spectrum of the PRNU image (where the illumination gradient has been removed) is flat along the lines indicating no correlation from pixel to pixel, which is consistent with the $1 / \sqrt{ } \mathrm{N}$ smoothing observed, while the spectral shape matches that predicted by simulation of pixel boundary randomization at all frequencies where there is significant power (i.e. for scale length $<30$ pixels).

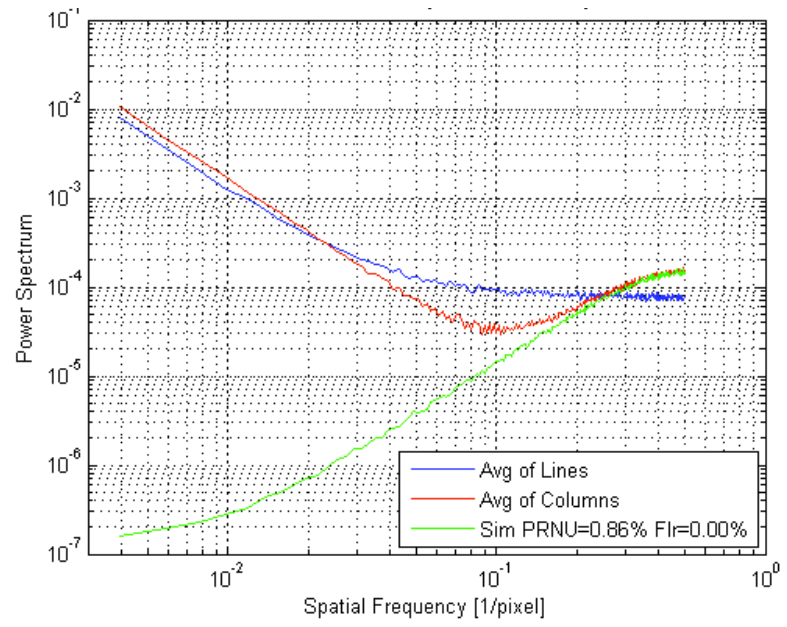

Fig. 10: For the raw flat field, power spectra are calculated for individual lines and columns then averaged to improve $\mathrm{S} / \mathrm{N}$. The low frequencies are dominated by the illumination gradient rather than sensitivity, while high frequencies must be due to CCD properties since the illumination is smooth. The marked difference between line and column directions suggests fundamentally different sources of PRNU.

The simulated spectrum in Fig. 11 (lower curve) is a prediction, not a fit. It is based only on the standard deviation of the PRNU image and the assumption that all PRNU variability is due to pixel boundary randomization. The close agreement with the measured column power spectrum indicates that it is very likely that almost all the power in the PRNU (on scales less than 30 pixels) is due to pixel area variations.
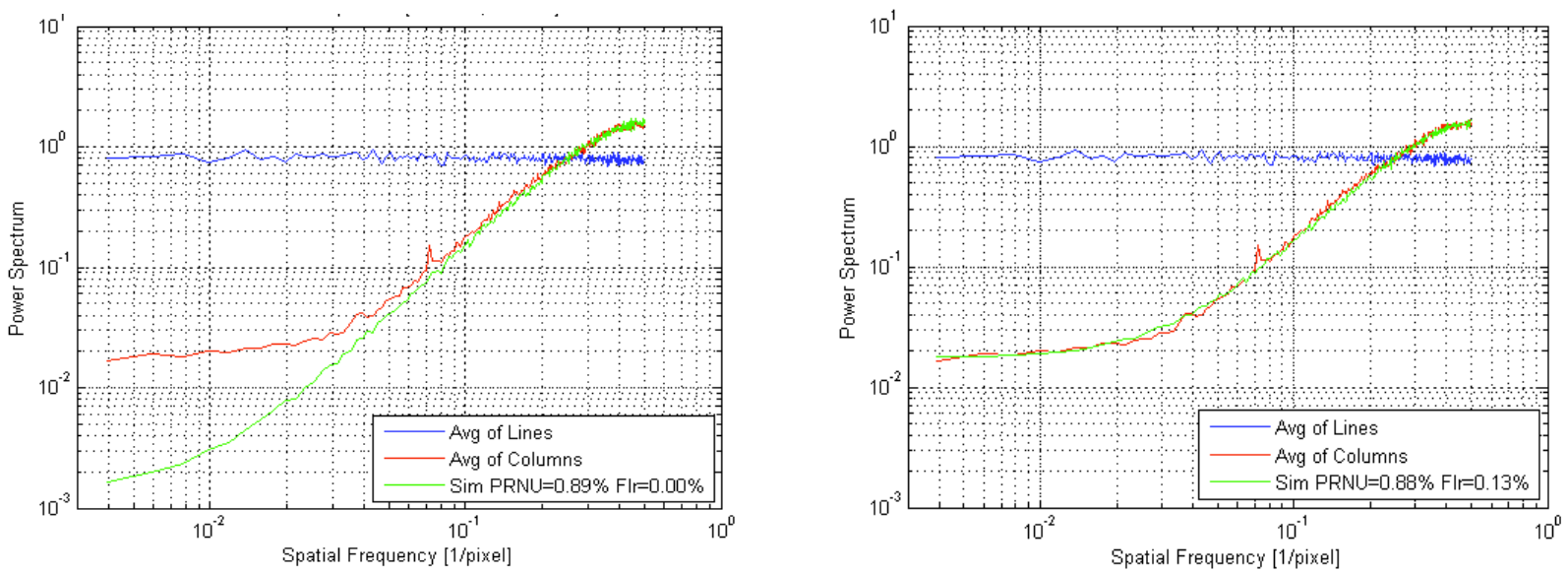

Fig. 11: When the raw flat is high pass filtered by dividing by a 9x9 boxcar (moving average), the difference between line and column power spectra becomes dramatic. The power spectrum of pixel size variation predicted from the standard deviation of the image is a very good fit to the column power spectrum at high frequencies (left plot). The right plot shows that an almost perfect fit is obtained when $0.13 \%$ white noise is added: the component due to pixel size must be reduced only slightly to $0.88 \%$. 
By arbitrarily repartitioning the $0.89 \%$ standard deviation of the PRNU image into $0.13 \%$ white noise and $0.88 \%$ due to pixel size variation, an almost perfect fit to the observed spectrum is obtained (right plot in Fig. 11). The simplest interpretation is that $0.88 \%$ PRNU is due to pixel size variation and that QE variations are uncorrelated from pixel to pixel and thus have a white spectrum, or at least the component left behind by boxcar filtering is white. While it is possible that the QE variation could be biased to high frequencies it would have to mimic the spectral shape of the pixel size variation to have any significant contribution while maintaining such a good fit. We plan to resolve this ambiguity by measuring the variation in integrated signal from a one pixel wide spot as it is scanning across the CCD, comparing results with and without flat fielding.
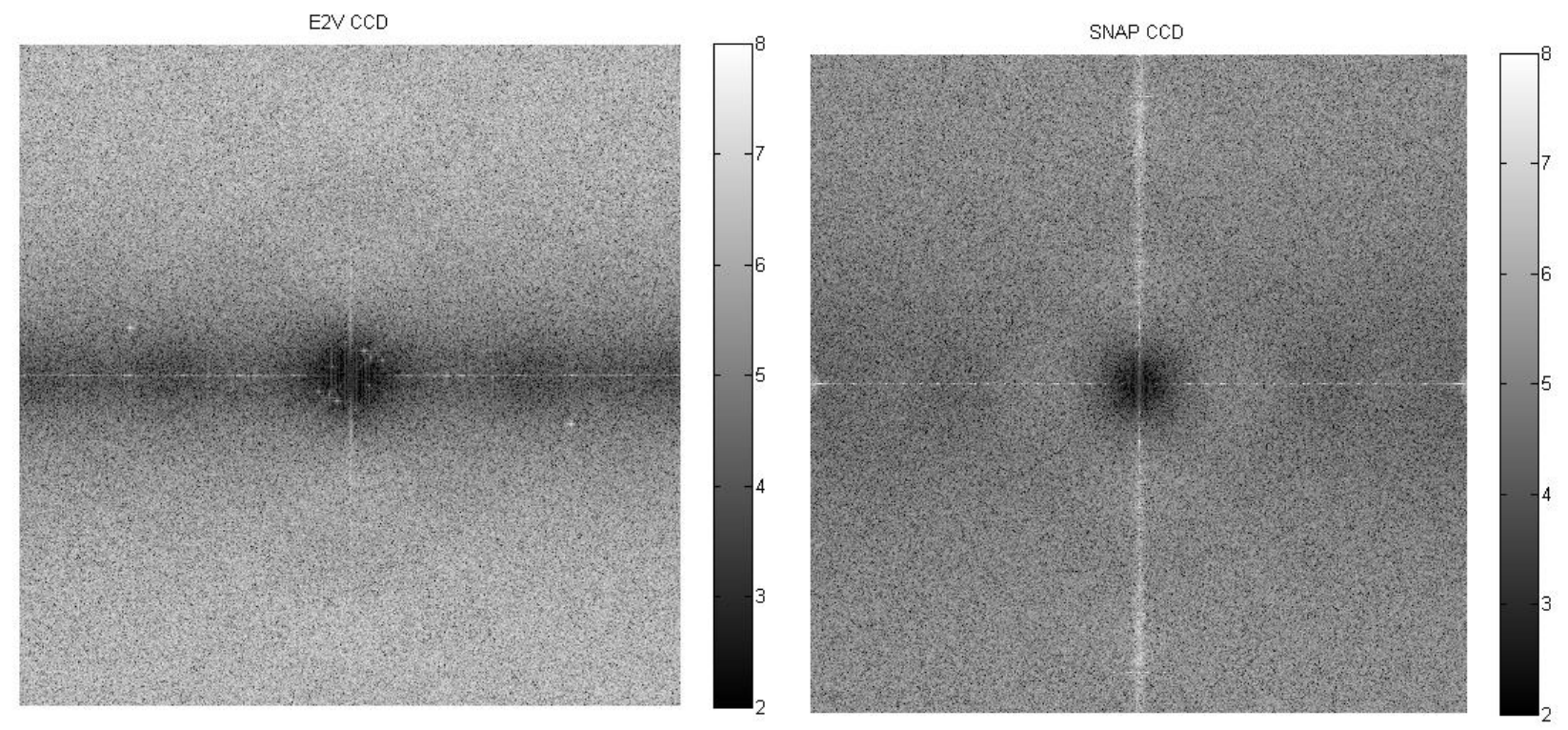

Fig. 12: 2D fourier transform of PRNU images from the e2v and SNAP CCDs presented in this paper. Zero frequency is at the center with frequency $= \pm$ Nyquist at the edges. Frequency scales are linear, while intensity is logarithmic. Bright points on the axes are due to entire rows or columns exhibiting width variations periodically. The faint dark horizontal band spanning the entire width at low frequencies is due to lack of energy due to random pixel height variation.
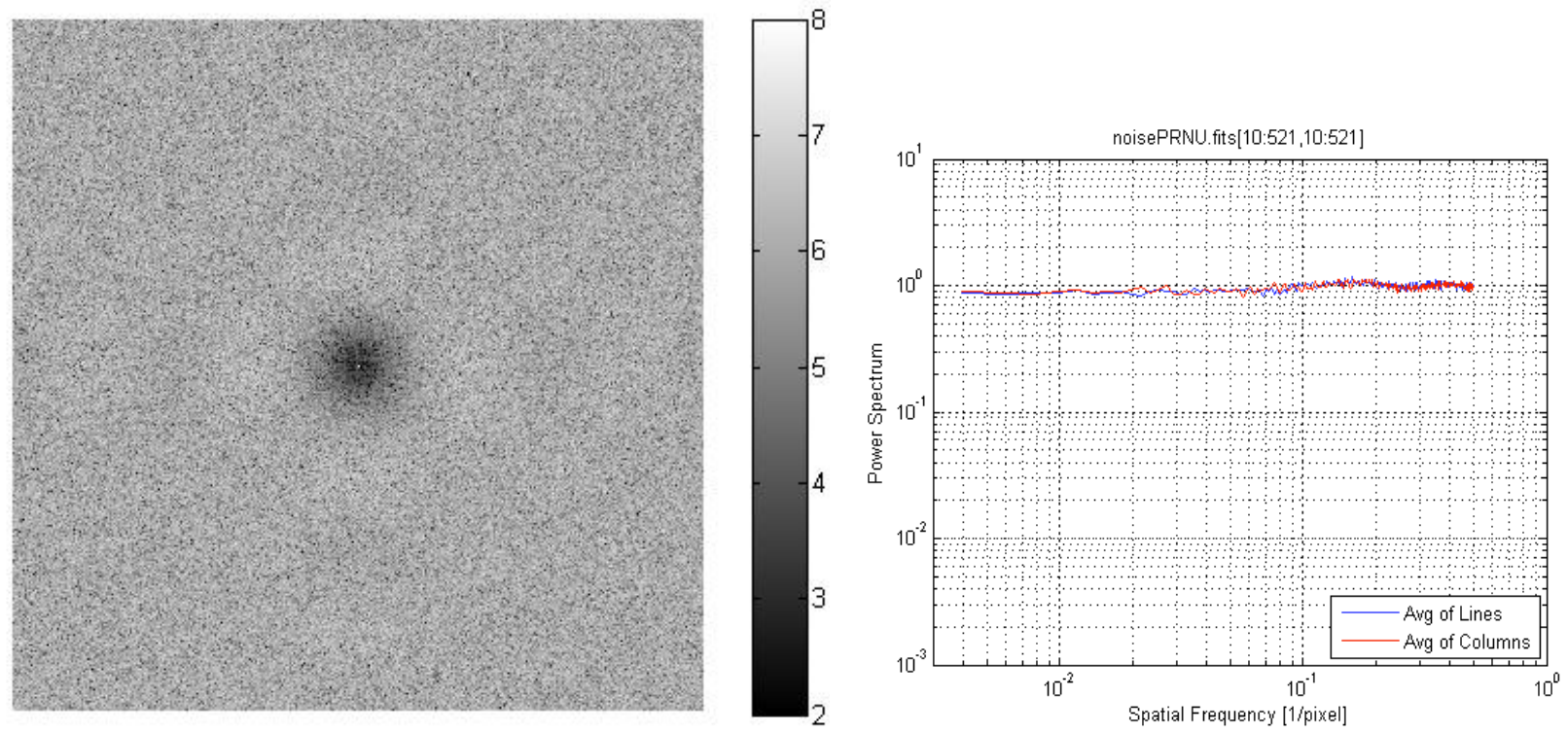

Fig. 13: 2D fourier transform (left) of a simulated 0.993\% PRNU image formed by dividing a 10,000 e- signal with Poisson noise by a 9x9 boxcar filtered version of itself. This suppression of low frequency content surrounded by slightly 
brightened rings is also seen in Fig. 12 showing that these are artifacts of the algorithm used to generate the PRNU

image. At right, the average 1D power spectrum of this simulated PRNU image is shown for comparison with Fig. 11.

If the pixel height variation (along columns) is uncorrelated with the pixel width variation (along rows) then the power spectrum along rows will be swamped by what appears to be a random signal due to pixel height variation, so that no signature of width variation would be visible in the spectrum along the rows. Similarly, it is plausible that the pixel width variation accounts for the white noise floor observed in the column spectrum. However, 2D fourier tranforms of the PRNU images (Fig. 12) for the e2V CCD, and for the SNAP CCD described below, do not support this or at least the effect is too weak to be seen against the artifacts produced by the algorithm for generating the PRNU image (Fig. 13).

\section{PRNU FOR SNAP FLIGHT CCD}

The CCDs developed by LBNL are different in almost every respect to the e2v CCD presented above, yet it will be shown that random pixel size variation along the columns is still the predominant source of PRNU. The e2v CCD is a conventional n-channel device collecting electrons, while the LBL CCDs are p-channel devices collecting holes. The $\mathrm{e} 2 \mathrm{v}$ is thin to minimize lateral diffusion of photoelectrons generated in the field free zone near the back surface, whereas the LBL CCD is 20 times thicker (to extend the red cutoff), is made on high resistivity material, and has a backside electrode which creates an electric field throughout the entire volume to collect charge with minimal lateral diffusion. e2v CCDs are made in their own production facility using step and repeat printing (stitching), while the LBL CCDs are fabricated at the DALSA foundry where 1:1 full-wafer mask projection is used.

At $10.5 \mu \mathrm{m}$ the pixel size of the $3500 \times 3500$ SNAP flight CCD is about one 3rd that of the e2v CCD so one might expect greater PRNU variations from any of the known causes. Therefore it was surprising to find that the standard deviation of the total PRNU for the SNAP CCD is only half that of the e2v CCD. More in line with expectations is the fact that the patterns due to width variation of entire lines or columns is stronger and is clearly visible in the PRNU image (Fig. 14).

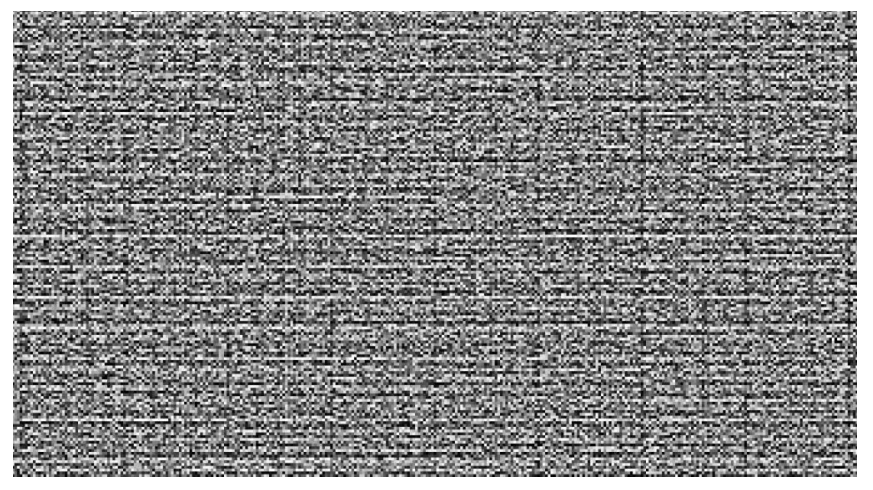

Fig. 14: The SNAP CCD flat field exhibits only $0.46 \%$ PRNU, half that of the e2v CCD. Periodic line and column patterns are large enough to be seen against the lower random component. A $325 \times 180$ pixel region is shown.

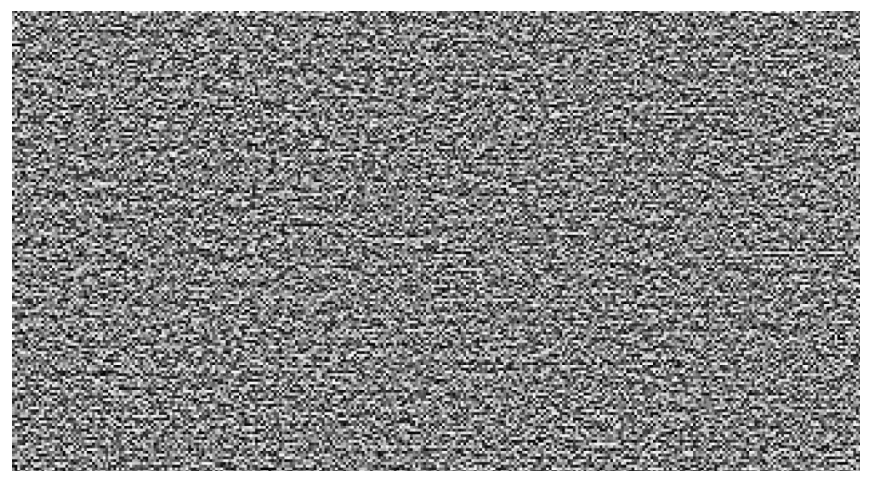

Fig. 15: The periodic line and column patterns are heavily suppressed, but not completely removed, by subtraction of the average line from each line and by subtraction of the average column from each column. The same $325 \times 180$ pixel region as in Fig. 14 is shown. 
Like the e2v, the standard deviation (along lines) drops more rapidly for the SNAP CCD, when smoothing along columns, than does the standard deviation (along columns) when smoothing along lines. This provides evidence that pixel size variation along columns is again the largest source of PRNU, however the rate at which the standard deviations decrease is less than $1 / \mathrm{N}$ and $1 / \sqrt{ } \mathrm{N}$ respectively (Fig. 16).

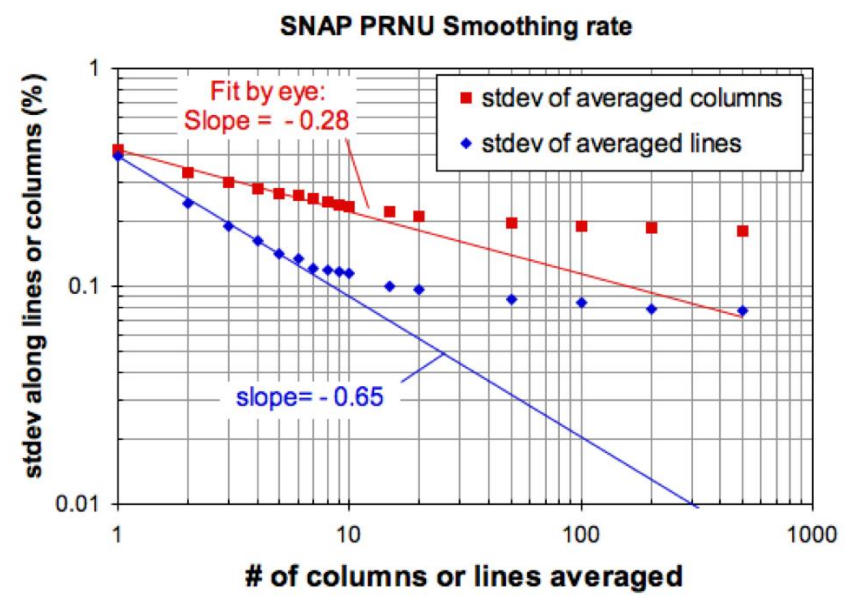

Fig. 16: While the smoothing rate for the SNAP CCD is twice as fast along columns as along lines (similar to the e2v), the actual rates are lower than expected. The floor caused by fixed patterns is higher too.

The stronger fixed pattern contribution causes the standard deviation on both axes to reach a floor sooner. Could fixed patterns also be responsible for the lower smoothing rate? To test this, we suppressed the fixed patterns by subtracting the average line from each line and the average column from each column of the PRNU image to produce Fig. 15.

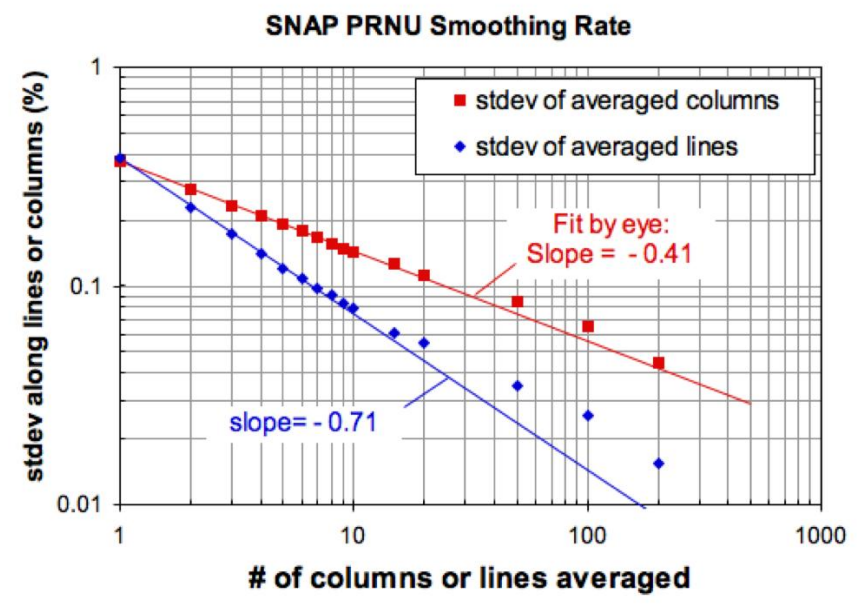

Fig. 17: Suppression of patterns caused by variation in width of entire lines and columns not only suppresses the floor in the smoothing curves but also increases the slopes. The fact that the slopes are still less than expected for uncorrelated and anti-correlated random patterns suggest that the pattern was incompletely removed due to some amplitude variation in across the CCD.

This had the effect of suppressing the floor in the smoothing curves (Fig. 17) and increasing the smoothing rate everywhere, however there would appear to be some residual line/column pattern that has not been corrected since the smoothing rates, while closer, are still not $1 / \mathrm{N}$ and $1 / \sqrt{ } \mathrm{N}$. These ideal smoothing rates will only be seen if the PRNU structure is fully random (anti-correlated or uncorrelated from pixel to pixel, respectively). If the amplitude of the width variation of entire lines or columns is not quite constant across the image, the subtraction of the mean pattern will over or under-compensate in some places leaving a component which does not average away as rapidly. A hint of residual fixed pattern can be seen in the corrected image (Fig. 15), so this appears to be the correct explanation. 
Unlike the smoothing curves, Fourier analysis measures random and periodic patterns separately. Fig. 18 (left panel) shows a the fit to the PRNU image without suppression of the fixed pattern, while the right panel shows that the fit is almost identical when line column averages have been subtracted to suppress the fixed pattern.
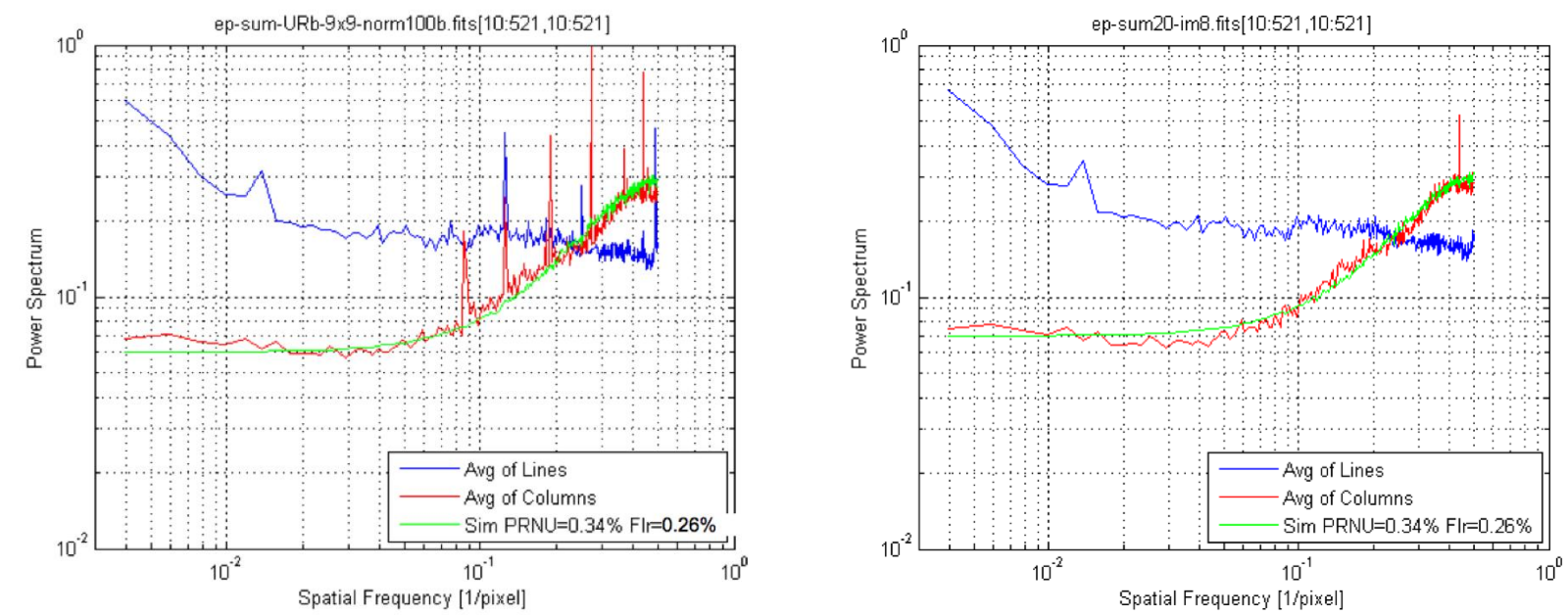

Fig. 18: A combination of $0.34 \%$ pixel size variation and $0.26 \%$ white noise, presumed to be due to QE variation, is a good fit to the column power spectrum. The line power spectrum shows slightly more structure than the e $2 \mathrm{v}$ line spectrum, but is qualitatively similar insofar as being much flatter than the column power spectrum. The periodic components in the spectra at left are due to the width variation of entire lines and columns, also seen in the PRNU image and line and column averages. The spectra at right show that subtraction of average lines and columns removes most of the energy at single frequencies, but does not affect the underlying spectrum caused by random variations. The periodic component at $\sim 2.3$ pixel period was not completely removed, perhaps because it is variable in amplitude across the CCD.

\section{DISCUSSION}

In summary, the predictability of the power spectrum of PRNU caused by random pixel boundary displacement allows us to decompose the PRNU pattern into a white spectral component, presumed to be due to random QE variations, and a component biased to high frequencies due to the anti-correlation in pixel area caused by boundary offsets. We find that the random pixel size variation along columns dominates the variation in $\mathrm{QE}$, as well as patterns caused by variation in the width of entire lines (Table 1).

\begin{tabular}{|c|c|c|c|}
\hline PRNU from: & $\begin{array}{c}\mathrm{e} 2 \mathrm{v} \\
30 \mu \mathrm{m} \text { pixel }\end{array}$ & $\begin{array}{c}\text { SNAP } \\
10.5 \mu \mathrm{m} \text { pixl }\end{array}$ & $\begin{array}{c}\text { SNAP } \\
\text { Patterns } \\
\text { suppressed }\end{array}$ \\
\hline Shot noise & 0.03 & 0.10 & 0.10 \\
\hline QE & 0.13 & 0.24 & 0.24 \\
\hline Random Size & 0.88 & 0.34 & 0.33 \\
\hline Periodic size & 0.02 & 0.18 & suppressed \\
\hline Quadrature sum & 0.890 & 0.464 & 0.420 \\
\hline Measured rms & 0.890 & 0.460 & 0.425 \\
\hline
\end{tabular}

Table 1: PRNU for the e2v and SNAP CCDs studied is dominated by pixel size variation along columns even though they are fabricated using radically different processes. As might be expected for the much smaller pixels, QE variation contributes more to the SNAP CCD than for the e2v. The SNAP CCD exhibits about half the overall PRNU due its much lower random size variation. The greater variation in width of entire lines or columns may prove correctable by matching the pixel size to the mask writer resolution.

No pixel width variation is detected in power spectra along rows. It may be present but at a level to low to detect in the presence of the pixel height variation which appears as a strong white noise component in the row direction. 
A greater QE variability from pixel to pixel in the SNAP CCD is to be expected given the factor of 8.2 in pixel area. Stronger periodic errors in line and column widths were expected for the smaller SNAP pixels, but the significantly lower random pixel height variation was a surprise given that the clock voltage swings were similar.

An examination of CCDs on the same wafer with different orientations shows that the periodic patterns are not related to the CCD row and column directions but to the raster direction of the mask writer.

The peak amplitude of the systematic line or column width error is on the order of $50 \mathrm{~nm}$ which is larger than the typical $5-8 \mathrm{~nm}$ quantization of the beam position in the mask writer, so spatial quantization errors and Differential Non-Linearity in the DAC driving the beam position seem unlikely causes of line width errors. However the beam that writes the mask optically scans about a one millimeter wide swath while the mask is driven by a scan table on the other axis, returning to scan the next $\sim 1 \mathrm{~mm}$ wide swath to build up a wafer scale mask (Fig. 19). Errors in the positioning of the mask by the $\mathrm{XY}$ stage are the most plausible cause for the periodic line and column width errors seen in the flat fields.

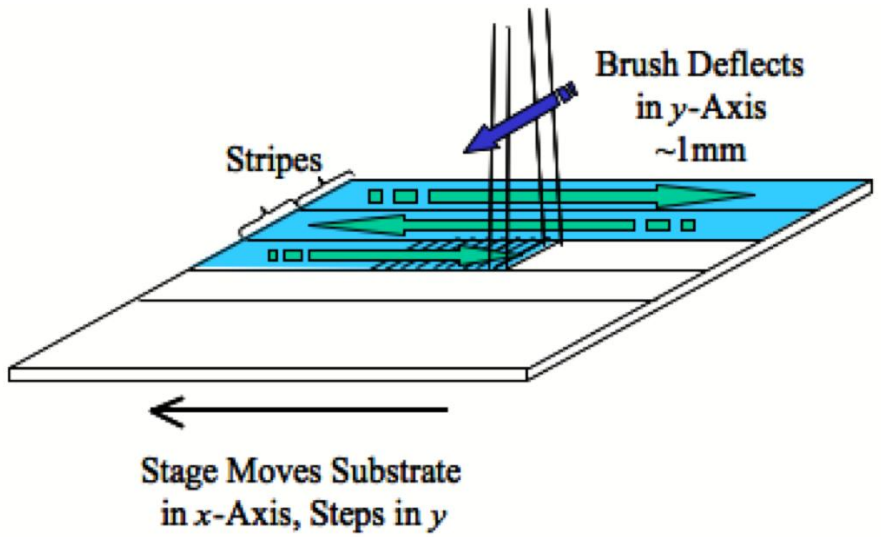

Fig. 19: Beam scanning pattern for mask writer. The "brush" consists of 32 beams at 5-8nm spacing with independent intensity control. Each brush stroke is offset by 16.5 times the beam separation to provide an interlaced scan. Errors in $\mathrm{XY}$ scan table positioning would appear to be responsible for the systematic line and column width patterns seen in the flat fields.

Elimination of the periodic line and column width variation in the SNAP CCDs would reduce the total PRNU (for the case where shot noise is negligible) from $0.453 \%$ to $0.416 \%$.

\section{FLAT FIELDING PROPOSAL}

If the PRNU image is used at full spatial resolution to normalize pixel values it will introduce a $\sqrt{ }(0.342+0.182)=0.38 \%$ rms error in an attempt to correct for only $0.24 \%$ QE variation. To avoid this, the PRNU image should be smoothed in the column direction (only) using a filter having the inverse shape to the power spectrum for random pixel boundary offsets, or at least some filter shape which rolls off at the corner frequency formed by the white noise and the pixel size variation, about 20 pixels for the SNAP CCD.

Since, for this experiment, the PRNU image was made by dividing the flat field by a 9x9 boxcar-smoothed version of itself, there will be little information left in the column direction after this smoothing. Clearly the illumination used to generate the raw flat field needs to be flatter than in our experimental setup so that a larger boxcar can be used to create the PRNU image: this will allow mid spatial frequencies to be corrected. Note however that even with a 9x9 boxcar, the column-wise-smoothed PRNU correction is still applied at full spatial resolution along the rows and thus has some merit.

The discussion so far has neglected large-amplitude QE drop-outs such as dust spots. Since pixel size variations produce a very tight Gaussian distribution (Fig. 20), outlying values as small as a few percent can be clearly recognized as predominantly QE variation. These should be corrected at the full spatial resolution of the PRNU image: precise correction of large QE drop outs will outweigh the slight error introduced by the underlying pixel area variation. When correcting for small scale PRNU, pixels containing outlying values should be replaced by unity (the mean value of PRNU, by definition) prior to smoothing, because they will have already been corrected and because we do not want the smoothing to smear the QE drop-outs over a larger area. 


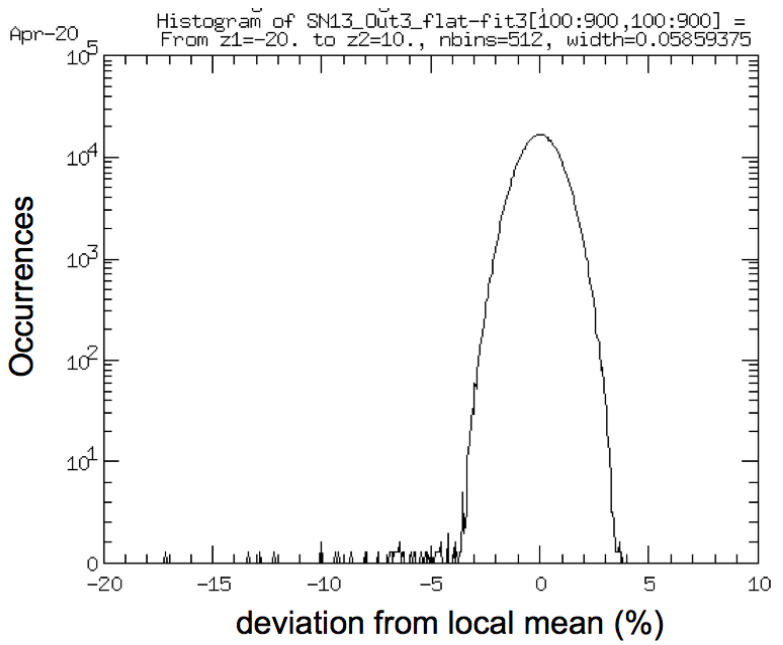

Fig. 20: Histogram of PRNU deviations for the e2v CCD. Since this has an ideal Normal Distribution without tails, values more than 4\% below the mean are clearly due to QE drop outs. Note that there are no outliers on the high side.

Finally it should be noted that for the simplistic case where signal is integrated over a rectangular aperture $\mathrm{X}^{*} \mathrm{Y}$ pixels, with no flat fielding at all on spatial scales shorter than 20 pixels, the random error due to pixel size variation goes as $0.34 \% /(\mathrm{Y} \sqrt{ } \mathrm{X})$ for the SNAP CCD while random QE variation (the white noise floor in the PRNU spectrum) will scale as $0.24 \% / \sqrt{ }(\mathrm{XY})$. Thus in the simple case of aperture photometry with a $2 \times 2$ aperture (an illustrative example, not the actual technique proposed for SNAP) the residual QE error, would be $0.12 \%$ if no PRNU correction is applied. Coincidentally the error due to pixel size variation would be the same, $0.34 /(2 \sqrt{2})=0.12 \%$, if the PRNU was applied at full spatial resolution. Smoothing along the columns before applying the PRNU should provide an improvement over this, which we have yet to quantify.

SNAP will observe all supernovae at four telescope pointings, offset by several pixels in each direction, to remove cosmic rays and cosmetic defects. Combining these four images will provide another factor two reduction in the errors due to uncorrectable PRNU, so that the final residual errors should make only a small contribution to the overall photometric error.

\section{REFERENCES}

[1] Janesick, J.R., [Scientific Charge Coupled Devices] SPIE Press (2001)

[2] Bebek, C. J., "Dark Energy and the SNAP Space Mission" Proc. IEEE Nuclear Science Symposium (2007) 